Artigo de Revisão

\title{
Intervenção grupal com professores com síndrome de burnout: uma revisão integrativa
}

Group intervention with teachers with burnout syndrome: an integrative review

Luciana de Araújo Mendes e Silva ${ }^{1}$, Vitória Regina de Morais Cardoso Rodrigues ${ }^{1}$, Alessandra Faleiros Silveira ${ }^{1}$, Cleria Maria Lobo Bittar ${ }^{1}$.

${ }^{1}$ Universidade de Franca - UNIFRAN, São Paulo, SP- Brasil

Submissão: 08/08/2016

Aceite: 26/09/2016

laraujo3@yahoo.com.br

\section{RESUMO}

Justificativa e objetivos: A profissão docente é considerada uma das mais estressantes e o prolongamento da situação de estresse pode causar a Síndrome de Burnout.Trata-se de uma revisão integrativa que objetivou levantar as produções científicas que ressaltam a importância de intervenções grupais como estratégias para promover enfrentamento e/ou prevenção do adoecimento causado pela síndrome. Conteúdo:A base de dados utilizada foi a SCIELO sendo a amostra constituídade 01 artigo obtido a partir dos critérios de inclusão e exclusão adotados na pesquisa. Evidenciou-se que os estudos realizam levantamentos sobre: fatores de risco da Síndrome de Burnout; prevalência da síndrome em docentes, conhecimento da síndrome pelos docentes e estratégias de enfrentamento. Entretanto apenas um estudo realiza ações interventivas.Conclusão:Destaca-se assim a necessidade de realização de pesquisas no país com ênfase na efetivação de intervenções grupais para prevenir ou reabilitar os docentes com síndrome de Burnout.

DESCRITORES: Esgotamento Profissional. Docentes. Estudo Clínico.

\section{ABSTRACT}

Background and objectives: Teaching is considered one of the most stressful professions and prolonged stressful situation can lead to burnout syndrome. The purpose of this integrative review was to identify the scientific productions that highlight the importance of group interventions as strategies to promote coping and / or prevention of illness caused by the syndrome. Contents:The database used was SCIELO and the sample was consisted of 01 article obtained from the criterion of inclusion and exclusion adopted in the research. It was evident that the studies conduct surveys about risk factors of burnout syndrome; prevalence of the syndrome in teachers, knowledge of the syndrome by teachers and confronting strategies. However, only does one study perform interventional actions. Conclusion:Thus, what stands out is the need of conducting research in the country with an emphasis on effective group interventions to prevent or rehabilitate teachers who were diagnosed with burnout syndrome.

KEYWORDS: Professional Burnout. Faculty. Clinical Trial.

\section{INTRODUÇÃO}


O trabalho não somente tem se relacionado à sobrevivência dos seres humanos, como também a produção e lucratividade, além de fonte de satisfação pessoal, por estar relacionado aauto realização e a manutenção das relações humanas. ${ }^{1}$

Vale aqui ressaltar que excesso de trabalho e as condições desfavoráveis para sua realizaçãopodem acarretar perda da qualidade de vida e até mesmo doenças incluindo nesse contexto, o estresse. ${ }^{2,3}$

O estresse ocupacional prolongado pode gerar um conjunto de sintomas, denominado Síndrome de Burnout. ${ }^{4} \mathrm{O}$ termo burnout, em língua inglesa, refere-se àquilo ou àquele que chegou ao seu limite, com grande prejuízo em seu desempenho físico ou mental. ${ }^{5}$

Atualmente a definição mais aceita dessa síndrome é baseada no modelo causal socialpsicológico referido por Maslach, Schaufeli e Leiter que a caracteriza em três dimensões: exaustão emocional, despersonalização e baixa realização pessoal no trabalho. ${ }^{5}$ A exaustão emocional (EE) é definida como a sensação de não se dispor de energia, seja mental, seja física, para continuar com as atividades laborais. Os sintomas psicossomáticos nessa dimensão acarretam o absenteísmo e o afastamento por problemas de saúde. ${ }^{6}$

A despersonalização (DE) ou Cinismo (CI), se caracteriza por atitudes desumanizadas e até mesmo irônicas e cínicas para com seus pares. Trata-se de dimensão defensiva aos sintomas experienciados. ${ }^{7}$

A dimensão reduzida realização pessoal (RRP) no trabalho, ou ineficácia, refere-se a um termo adotado que corresponde ao momento quando o trabalhador sente-se frustrado e insatisfeito com seu trabalho, se autoavaliando negativamente. ${ }^{8}$

Estudos revelam que os profissionais que se encontram mais predispostos a desenvolverem a Síndrome de Burnoutsão aqueles cujas funções se relacionam à prestação de serviços que lidam diretamente com o ser humano, dentre eles aqueles que trabalham com saúde, segurança e educação. ${ }^{9}$

A profissão docente é considerada mundialmente como uma das mais estressantes, com forte incidência de elementos que conduzem à Síndrome de Burnout. As exigências relacionadas ao exercício do magistério, à gestão escolar, à localização da escola e ao relacionamento do professor com a comunidade, têm trazido graves consequências à saúde desse profissional.$^{10}$

Laudos médicos em diferentes países referentes à licença de professores por motivos de saúde indicam maior incidência de doenças mentais em relação a outras doenças. ${ }^{2}$ Segundo esses autores, existe um forte indicativo de que essas doenças estariam relacionadas a atividades inerentes ao trabalho docente. A docência tem sido alvo de vários estudos e 
apresenta estressores psicossociais inerentes tanto às funções impostas aos profissionais quanto as relações sociais por eles vivenciadas. ${ }^{11,12}$ Tais estressorespodem interferir na saúde e, de forma persistente, podem levar à Síndrome de Burnout. ${ }^{12}$

Um estudo realizado em escolas de todos os estados do Brasil durante dois anos e meio, com uma amostra de 39 mil trabalhadores em educação, revelou que $48 \%$ dos educadores apresentavam algum sintoma de Burnout, 32\% dos participantes apresentavam baixo envolvimento emocional com o trabalho, $25 \%$ apresentavam exaustão emocional e $11 \%$ apresentavam despersonalização. ${ }^{13}$ Pesquisas realizadas nos Estados Unidos e na Holanda em cinco diferentes setores profissionais corroboram esses resultados ao constatar que o ensino foi caracterizado por altos índices nas três dimensões de Burnout. ${ }^{14}$

Todavia, mesmo sendo os professores propensos a desenvolverem Burnout, poucos possuem conhecimento dessa síndrome e se previnem em relação a seus efeitos. ${ }^{4}$ Alguns estudos internacionais têm trazido amplos modelos conceituais para a síndrome. ${ }^{14}$ Porém, consideram que é necessário, a partir das descrições desses estudos, buscar novas perspectivas em relação a intervenções para alivio a Burnout. ${ }^{14}$ Também alguns estudos brasileiros apontam a urgência de pesquisas que discutam estratégias de enfrentamento pelos docentes e ao mesmo tempo efetivem intervenções, porém apontam que tais iniciativas ainda são escassas e muitas vezes ineficazes. ${ }^{15,16}$

Relato de uma experiência com estratégias para intervenção utilizadas com professores menciona que se têm utilizado grupo focal ou grupo de discussão, além de conferências para grandes grupos. O autor ressalta que na Secretaria Municipal de Educação da Prefeitura de Curitiba, tem-se trabalhado, desde 1997, com sessões/encontros especialmente voltados para a promoção da saúde de professores, com especial atenção a Síndrome de Burnout. O autor salienta também que a promoção da saúde docente é um campo com múltiplos desafios, mas que as recompensas das intervenções realizadas justificam todo o esforço envolvido em estratégias desenvolvidas em pequenos grupos e posteriormente levadas a grupos maiores. ${ }^{10}$

Diante desse contexto, após busca na literatura por artigos relacionados à Síndrome de Burnout em docentes, verificou-se que raros são os estudos que se relacionam a intervenções grupais realizadas com o propósito de promover ações que visem à prevenção da síndrome. Pelo exposto e considerando-se o que prescreve a Política Nacional de Promoção de Saúde o Ministério da Saúde no sentido de que o processo saúde-adoecimento pode ser resultado dos modos de produção do trabalho, exigindo, desse modo uma análise do conhecimento dos sintomas das doenças, mas também a oferta de condições, e ações interventivas para efetividade da melhoria das condições de saúde, o objetivo dessa revisão integrativa foi 
levantar as produções científicas que relatassem experiências de intervenções grupais como estratégias para promover enfrentamento e/ou prevenção do adoecimento causado pela Síndrome de Burnout. ${ }^{17}$

\section{MÉTODOS}

O presente estudo constitui-se de uma revisão integrativa da literatura brasileira com o propósito de reunir e sintetizar conhecimentos pré-existentes sobre intervenções em grupo para promover a saúde de docentes com Síndrome de Burnout.

O procedimento inicial desse estudo foi a definição da questão norteadora da pesquisa que foi a seguinte: "Quais são as produções científicas relativas às intervenções em grupo, realizadas com o propósito de promover ações que visem o enfrentamento e/ou a prevenção da Síndrome de Burnout em docentes?"

A etapa de coleta de dados ocorreu no mês de dezembro de 2015 após a definição da base de dados e dos critérios de inclusão e exclusão. A base de dados escolhida foi a Scientific Electronic Library Online (SciELO) que é uma biblioteca eletrônica que abrange uma coleção selecionada de periódicos científicos brasileiros e da América Latina.

Os critérios de inclusão adotados admitiam somente os artigos de estudos realizados no Brasil que estivessem disponibilizados na íntegra, publicados em português, inglês ou espanhol no período de janeiro de 2000 a dezembro de 2015, cujos resultados fossem de pesquisas de revisão ou de campo. Foram considerados inicialmente os artigos com os termos registrados nos Descritores em Ciências da Saúde(DeCS). "Burnout" and"Teacher" e em seguida cruzado com "Intervention"1 que poderiam estar tanto no título, resumo e texto completo do artigo.

\section{RESULTADOS E DISCUSSÃO}

Do cruzamento das duas primeiras palavras-chave ("Burnout" and "Teachers") foram encontrados 34 artigos realizados no Brasil, sendo 31 no idioma português, dois em inglês e dois em espanhol.

Dezesseis artigos faziam alguma referência à intervenção com foco no indivíduo, no grupo ou na organização. Eles apresentavam discussões sobre a importância de intervenções 
grupais como estratégias para promover enfrentamento e/ou prevenção do adoecimento causado pela Síndrome de Burnout, os outros 18 tratavam de Burnout docente, mas não faziam nenhuma referência as intervenções e sua importância. Desses 16 artigos, 13 eram pesquisas de campo e três de revisão. Quanto aos estudos de revisão, um deles discute sobre as características da profissão docente e a ocorrência de Burnout apresentando dados do Brasil e de outros países. Enumeram variáveis associadas à síndrome e apontam que tais conhecimentos são indispensáveis para desenhar programas de intervenção que vão ao encontro das necessidades profissionais na docência. ${ }^{18}$

No segundo artigo de revisão são discutidos os fatores de risco associados à docência traçando um paralelo entre o prazer e a dor na docência e mencionam que o conhecimento sobre tais fatores possibilita condições de planejar e implementar ações intervencionistas e preventivas nos ambientes de trabalho, para o aumento da qualidade de vida e a construção de um ambiente escolar saudável. ${ }^{6}$

No terceiro artigo de revisão são descritas as causas e consequências da síndrome e se propõem medidas saneadoras como: diagnóstico do indivíduo, do local de trabalho e das pessoas relacionados à sua profissão; implementação de uma diversidade de estratégias de intervenção que incluem desde formação, atendimento médico especializado, reuniões grupais, etc.; capacitação profissional; atividades para trabalhar a comunicação e relações interpessoais; realização de técnicas coordenadas por profissional psicólogo; e realização de oficinas com temáticas variadas relacionadas ao cotidiano da profissão. ${ }^{19}$

Já os 13 estudos restantes, apesar de não trazerem a temática da intervenção nos objetivos ou mesmo nos resultados dos estudos, esta aparece nas considerações finais como algo desejável para o enfrentamento ou a prevenção da síndrome.

Contudo ao cruzar a terceira palavra-chave "intervention" apenas um artigo atendeu os critérios adotados, pois foi o único que efetivamente tratou-se de um estudo que descreveu uma estratégia de intervenção de enfrentamento da síndrome com docentes.

$\mathrm{Na}$ análise do único artigo encontrado com realização de intervenção grupal em relação a Burnout docente pode-se perceber que os autores justificam a relevância de seu estudo ao mencionarem que existe produção científica sobre a relação professor-estresseburnout, porém na visão desses pesquisadores persiste uma lacuna relacionada a essa problemática no que diz respeito a efetivação de estudos que realizem/proponham programas de intervenção. ${ }^{20}$ Esses achados são coincidentes com as conclusões de Hernandez et al. (2002) cujos dados coletados sugerem a necessidade de programas de intervenções com 
professores, contudo esses autores também citam que os programas de intervenção tem sido escassos ou praticamente inexistentes. $^{21}$

Estudo realizado em 2006 como seu objetivo a realização de grupos operativos numa perspectiva de intervenção para a mudança aponta benefícios desse método interventivo ao declarar que ele propicia a partilha de experiências profissionais, diminui o isolamento, oferece apoio social pessoal e profissional dos docentes. ${ }^{20}$ Afirmação reforçada em outro estudo ao considerar que a manutenção de apoio social adequado dentro e fora do ambiente de trabalho previne o aparecimento de Burnout. ${ }^{22}$

O fundamental na tarefa grupal num grupo interventivo é a busca de soluções para situações consideradas difíceis e até mesmo imutáveis promovendo uma reflexão sobre a realidade e necessidade de mudanças. $\mathrm{O}$ processo grupal inclui momentos para discussão sobre as condições de trabalho e se encerra com a construção de uma estratégia operativa para enfrentamento das causas e efeitos da Síndrome de Burnout. ${ }^{20}$

O estudo em análisecontou com a aplicação doinventário denominado Maslach Burnout Inventory(MBI) que verifica a ocorrência dos sinais de Burnout. ${ }^{20}$ Tal instrumento foi aplicado antes e após a intervenção tanto para o grupo controle quanto para o experimental que participou de nove encontros com debate sobre a percepção em relação à profissão docente. Após a realização dos encontros foi refutada a hipótese de melhoria de sintomas ao grupo experimental atestada pelos pesquisadores, ao contrário houve aumento nos sinais das dimensões da síndrome. Após a análise do conteúdo, suas significações, convergências e divergências os autores inferiram que inicialmente o grupo teve discussões superficiais, com o passar do tempo os diálogos se tornaram mais realistas despertando ansiedade o que justifica o aumento nos níveis de estresse. Mas ao mesmo tempo em quetal ansiedade tomou conta dos participantes o apoio social dos colegas supriu a falta do apoio institucional o que é corroborado por outro estudo que atesta que o sentimento de união e respeito entre colegas melhora a vida laboral. ${ }^{23}$

Com a finalização dos encontros o grupo saiu de uma visão alienante de sua profissão para uma atitude investigadora e em busca de mudança enquanto que o grupo controle continuou com uma visão inadequada de sua realidade profissional. Mesmo tendo elevado o nível de Burnout no grupo experimental, criou-se uma maior consciência e conhecimento de estratégias de enfrentamento através das vivências no grupo. Acreditam os autores do artigo em discussão, que o aumento na quantidade de encontros poderia consolidar as ações de enfrentamento e diminuir os níveis de ansiedade. A pequena quantidade de encontros também foi um aspecto avaliado em outro estudo de intervenção grupal não presente na base de dados 
em análise (encontrado em outra base ao longo da construção do presente artigo)sendo que mesmo considerando-se a pequena quantidade de encontros, o estudo trouxe segundo os participantes diversos aspectos positivos relacionados à prevenção/controle da síndrome de Burnout. $^{24}$

Entretanto apesar dos benefícios das estratégias interventivas,a criação e sistematização de grupos operativos dependem das instituições de ensino que deveriam colocar tais ações como prioridade tendo em vista que os benefícios se estendem além dos grupos operativos influenciando os alunos e a comunidade escolar como um todopois "Sem a docência saudável não existe e nem existirá uma sociedade culta e sadia". 20,25

\section{CONCLUSÃO}

A presente revisão evidenciou a preocupação dos pesquisadores em apontar os fatores de risco relacionados à ocorrência da Síndrome de Burnout; a prevalência da síndrome em discussão entre os docentes; o perfil/caracterização sóciodemográfica e ocupacional dos acometidos. Os conhecimentos presentes nas pesquisas revisadas de acordo com os autores apontam a necessidade de intervenções bem como podem contribuir para despertar as mesmas com o objetivo de minimizar as consequências desse agravo aos profissionais e consequentemente beneficiar a comunidade escolar.

Pode-se perceber que embora os estudos apontem para a necessidade de serem adotadas ações interventivas para a prevenção do Burnout, apenas um destes estudos satisfez plenamente o critério de busca, que objetivava ações grupais para prevenção deBurnoutem docentes. Tal fato caracteriza uma lacuna na produção científica brasileira em relação a estudos com aplicação prática de intervenções com docentes em relação a referida síndrome. Essa realidade sugere a necessidade de realização de estudos dessa natureza ainda que em pequenos grupos na busca de minimização das consequências do agravo em questão e com vistas à realização progressiva de trabalhos mais amplos.

\section{AGRADECIMENTOS}

À Coordenação de Aperfeiçoamento de Pessoal (CAPES) pelo apoio financeiro.

\section{REFERÊNCIAS}


1. Murta SG, Tróccoli BT. Avaliação de intervenção em estresse ocupacional. Psicol teor e pesqui 2004;20(1):39-47. DOI: http://dx.doi.org/10.1590/S0102-37722004000100006

2. Assuncão AA, Oliveira, DA. Intensificação do trabalho e saúde dos professores. Educ soc 2009;30(107):349-72.DOI: http://dx.doi.org/10.1590/S0101-73302009000200003

3. Benevides-Pereira AMT. O burnout docente e seu reflexo no ensino. In: Anais do X Congresso Nacional de Educação - EDUCERE e III Encontro Sul Brasileiro de Psicopedagogia, 11;3, 2011,Curitiba: Pontifícia Universidade Católica do Paraná, 2011.

4. Benevides-Pereira AMT. O CBP-R em português: Instrumento para a avaliação do burnout em professores. In: Anais do IX Congresso Nacional de Educação - EDUCERE e III Encontro Sul Brasileiro de Psicopedagogia, 9;3, 2009, Curitiba: Pontifícia Universidade Católica do Paraná, 2009.

5. Trigo TR, Teng CT, Hallak JEC. Síndrome de Burnout ou estafa profissional e os transtornos psiquiátricos. Rev de psiquiatr clín 2007;34(5):223-33.DOI: http://dx.doi.org/10.1590/S0101-60832007000500004

6. Andrade PS, Cardoso TAO. Prazer e Dor na Docência: revisão bibliográfica sobre a Síndrome de Burnout Saúde Soc2012;21(1):129-40. DOI: http://dx.doi.org/10.1590/S010412902012000100013

7 Batista JBV. Carlotto MS, Coutinho AS, et al. O ambiente que adoece: condições ambientais de trabalho do professor do ensino fundamental. Cad Saúde Coletiva 2010;18(2):234-41. DOI: http://dx.doi.org/10.1590/1414-462X201500020001

8. Carlotto MS. Síndrome de Burnout em professores: prevalência e fatores associados. Psicol Teor Pesqui 2011;27(4):403-10. DOI: http://dx.doi.org/10.1590/S0102-37722011000400003

9. Levy, GCTM, Nunes Sobrinho FP, et al. Síndrome de Burnout em professores da rede pública. Prod 2009;19(3):458-65. DOI: http://dx.doi.org/10.1590/S010365132009000300004 
10. Amorim CAA. Formação Continuada e a promoção da saúde do professor. In: Anais do VIII Congresso Nacional De Educação - Educeree III Congresso Ibero-Americano Sobre Violência Escolar, 8; 3, 2008, Curitiba: Pontifícia Universidade Católica do Paraná, 2008.

11. Batista JBV,Carlotto MS, Coutinho AS, et al. Prevalência da Síndrome de Burnout e fatores sociodemográficos e laborais em professores de escolas municipais da cidade de João Pessoa, PB. Rev Bras Epidemiol 2010;13(3):502-12. DOI: http://dx.doi.org/10.1590/S1415$790 \times 2010000300013$

12. Carlotto MS. A síndrome de Burnout e o trabalho docente. Psicol estud2002;7(1):21-9. DOI: http://dx.doi.org/10.1590/S1413-73722002000100002

13. Codo W (org.). Educação e Carinho. Petrópolis: Vozes/Brasília, DF: CNTE Universidade de Brasília, Laboratório de Psicologia do Trabalho.1999.

14. Maslach C, Schaufeli WB, Leiter MP. Jobburnout. Ann Rev clinPsychol 2001;52: 397422. DOI: http://dx.doi.org/10.1146/annurev.psych.52.1.397

15. Mazon V,Carlotto MS, Câmara S. Síndrome de Burnout e estratégias de enfrentamento em professores. Arq bras psicol 2008;60(1):55-66.

16. Santos MN, Marques AC. Condições de saúde, estilo de vida e características de trabalho de professores de uma cidade do sul do Brasil.Ciênc saúde coletiva 2013;18(3):837-46. DOI: http://dx.doi.org/10.1590/S1413-81232013000300029

17. Ministério da Saúde (BR). Secretaria de Vigilância em Saúde, Secretaria de Atenção à Saúde. Política Nacional de Promoção da Saúde. 3ª. ed. Brasília, DF. 2010. (Série B. Textos Básicos de Saúde).

18. Pocinho M, Perestrelo CX. Um ensaio sobre Burnout, engagement e estratégias de coping na profissão docente. Educ Pesqui 2011;37(3):513-28. 
19. Yurevna SI. Ayuda psicológica al pedagogo en la superación del síndrome de “incineración profesional”.Psicol Esc Educ 2011;15(1):161-7. DOI: http://dx.doi.org/10.1590/S1413-85572011000100017

20. Böck VR, Sarriera JC. O Grupo Operativo Intervindo na Síndrome de Burnout. PsicolEsc Educ 2006;10(1): 31-9. DOI: http://dx.doi.org/10.1590/S1413-85572006000100004

21. Hernández EG, Benevides-Pereira AM, Moreno-Jimenez JLGG. Prevenção e intervenção na síndrome de burnout. Como prevenir ou remediar o processo de burnout. In: BenevidesPereira AMT, editor. Burnout: quando o trabalho ameaça o bem-estar do trabalhador. 1. ed. São Paulo: Casa do Psicólogo. 2002. p.227-271.

22. Figueroa AEJ, Gutiérrez MJJ, Celis ERM. Burnout, apoyo social y satisfacción laboral en docentes. Psicol Esc Educ 2012;16(1):125-34.DOI:http://dx.doi.org/10.1590/S141385572012000100013

23. Benevides-Pereira AMB. O Processo de adoecer no trabalho In: Pereira AMB, organizador.Burnout: Quando o trabalho ameaça o bem-estar do trabalhador. São Paulo, SP: Casa do Psicólogo. 2002. p. 21-91.

24. Carlotto MSC. Prevenção da síndrome de burnout em professores: um relato de experiência. Mudanç psicol Saúde 2014;22(1):31-9. DOI: http://dx.doi.org/10.15603/21761019/mud.v22n1p31-39

25. Carneiro RM. Síndrome de Burnout: um desafio para o trabalho do docente universitário. [Dissertação]. Anapólis: Centro Universitário de Anápolis. 2010. 